\title{
The psychophysics of the pursuit oculomotor system
}

\author{
BRUCE BRIDGEMAN \\ University of California, Santa Cruz, California
}

\begin{abstract}
When a fixation point moves under a row of identical targets at a speed of one target for each flash of a strobe, smooth apparent movement of the targets is seen (the "picket-fence illusion"). When the fixation point is removed, the eye continues to pursue the apparent target movement. Pursuit continues through small changes in target configuration, but is interrupted by a change to a very dissimilar target (such as 1 vs. $x$ ) in the middle of a row. This new method, the "pursuitinterruption method," showed that large differences in the number of pixels in a line did not interrupt tracking if the end points of the line were preserved. Pursuit interruption by changes in line orientation (such as / vs. $\backslash$ ) corresponded to the orientation bandwidth of orientationsensitive cortical neurons. The maximum number of consecutive missing targets that does not interrupt pursuit depends on frequency of target presentation as well as on parameters of the pursuit system.
\end{abstract}

Sensory function is normally measured by presenting a stimulus to a subject and requiring a response such as a button press or a verbal report. Such measures assess only one output of the visual system; they do not examine information flow within the parts of the system that control oculomotor behavior.

Introspection might suggest that a single unified visual sense provides information for all visual functions, including control of pursuit eye movements. Recent evidence, however, indicates that different visual functions are controlled by different kinds of visual information. The functions can be separated with psychophysical techniques. With induced motion, for example, a target can be perceived to move in one direction while a saccadic eye movement follows it in the opposite direction (Wong \& Mack, 1980). This paradoxical result occurs when a large background pattern is displaced, causing an apparent motion of a small target in the opposite direction. At the same time, the target is displaced in the same direction as the background, in the direction opposite the apparent motion but too small to cancel the motion.

Another experiment (Bridgeman, Kirch, \& Sperling, 1981) demonstrated a double dissociation of information used for perception and for controlling visually guided behavior. When motion was induced in a fixed target because of the displacement of a surrounding frame, pointing to the target remained unaffected by frame position-a signal available to perception did not affect behavior. When the subject adjusted the displacement of the target in phase with frame displacement so that the target appeared to be fixed, pointing followed true target position even though the shift in position was not perceived. Thus,

This research was supported by a seed grant and a faculty research grant from the University of California, Santa Cruz. I thank Katherine Hanley for assistance in experimental work. Correspondence may be addressed to Bruce Bridgeman, Program in Experimental Psychology, Clark Kerr Hall, University of California, Santa Cruz, CA 95064. a signal that was available to guide motor behavior did not affect perception. This experiment showed that separate neural representations of visual space control the two outputs; one cannot be a subset of the other.

The possibility of segregating information inputs to one visual function or another raises the question of the visual information available to guide pursuit eye movements. This information may not be the same as that available to perception. Deubel, Findlay, Jacobs, and Brogan (1988) have isolated stimulus parameters used by the visual system to control saccadic eye movements; the goal of the present paper is to do the same for pursuit.

One possible method for studying pursuit would be to initiate tracking of a target, and then abruptly change the target's shape without interrupting its motion. A cessation of tracking would indicate that the system handled the new target as different from the old one. Pilot experiments showed that in this situation, tracking continues regardless of the change, however, presumably because all small targets have similar low spatial-frequency components, and these components can be tracked without interruption.

To isolate the information available to the pursuit mechanism, it was necessary to find a way to stimulate the system in isolation and measure its behavior. This is normally impossible because pursuit is part of a negativefeedback loop; the error correction inherent in the loop prevents isolation of the system's behavior. To examine the system, we have used a recently discovered stimulus based on positive feedback: motion signals are received only as long as the eye is moving, and pursuit is possible only if the system interprets successive stimulus samples as similar.

The stimulus and its accompanying motion illusion have been variously named the picket-fence illusion (Stoper, 1973) and sigma-OKN (Behrens \& Grüsser, 1978). To generate it, a row of bars or other repeating figures is illuminated with stroboscopic light. All bars are illumi- 
nated simultaneously. A continuously illuminated fixation point moves just below the row of bars at a speed of one bar for each interflash interval. A subject follows the fixation point; at each flash of the strobe, the row of bars is illuminated briefly, always in the same retinal location. The subject sees the row of bars seeming to move along with the fixation point. When the point is extinguished, the row of bars continues to appear to move smoothly as the subject follows it with pursuit eye movements even though there is no stimulus movement. The illusion was also discovered independently by Heywood (1973) and by Lamontagne (1973). In the present paper, the psychophysical motion illusion will be called the picket-fence illusion and the concomitant eye movements will be called sigma-OKN.

If the adequate stimulus for continuation of the movement is disturbed, eye movement should cease because there is no real motion and no structure at low spatial frequencies in the stimulus being pursued. (The lowest, or fundamental, spatial frequency orthogonal to the direction of tracking is at the repetition distance of the target characters, or 3 cycles/deg in our conditions.) The repeating identical targets give only an ambiguous motion signal if the smooth pursuit movement is disturbed. By presenting a sequence of flashes whose timing was modified with Gaussian noise, Adler et al. (1981) found that with a coefficient of variation (standard deviation/mean interval) of more than 0.36 , smooth eye movements and the picket-fence illusion broke down.

The need for continuity of a stimulus to enable continuity of eye movement provides a way of objectively measuring the similarity of two patterns according to the pursuit system's criteria, as the geometry of the figures in the row can be manipulated to discover the features that are important in maintaining pursuit. If one figure is repeated in the left half of a row and a very different figure in the right half, the eye may cease pursuit near the border between one figure and the other. The situation contrasts with that found during apparent motion of discrete stimuli. Kolers and Pomeranz (1971) found that apparent motion is seen less frequently if a stimulus changes its shape as it jumps. The nature of the change was not important, however, as the differences between various pairs of shapes did not affect the apparent motion.

With continuation of pursuit as the dependent variable and form of the tracked figures in stroboscopic illumination as the independent variable, experiments on the psychophysics of pursuit are possible.

\section{METHOD}

Pilot experiments were performed on an ordinary computer CRT terminal (Televideo 950). Because of the $60-\mathrm{Hz}$ raster method of display, all characters on the screen were strobed at $60 \mathrm{~Hz}$. First, a row of identical characters, for instance ls, was written on the screen:

1111111111111111111111111111111111111111111111111111111111111111111111111111111111111.

Then the experimenter/observer moved a finger across the screen at a speed of 80 characters $/ 60 \mathrm{~Hz}=1.33 \mathrm{sec}$ for a complete scan from left to right. When the finger was moved at the correct speed, an " 1 " would appear to drift across the screen, accompanied by a group of indistinct ls to its left and right. After some practice, the finger could be taken away following 10-20 characters of tracking, and smooth eye movements would continue nearly to the other side of the screen. Eventually, an experienced observer could begin pursuit voluntarily, without any moving stimulus. Pursuit generally broke down as the observer approached the end of a line, at a distance from the end that generally related to the "scan width," which we define as the number of characters that an observer's pursuit system uses to control the eye movements.

The pilot experiments showed that most observers could continue tracking through a minor change in the stimulus, such as "l" to " $i$,"

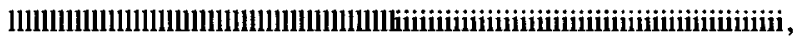

but their eyes would involuntarily come to a halt in mid-scan if the change involved a major change of the lines in the target figure, such as " $l$ " to " $\mathrm{x}$,"

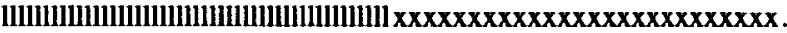

This technique, although simple to program, had two disadvantages. First, we were limited to the characters available on the terminal. Second, the row length was only 80 characters-not enough to allow all subjects to develop a stable, saccade-free pursuit, and to change characters while still showing a measurable disruption in pursuit before approaching the end of a line.

To overcome these difficulties, we moved to an Apple Macintosh Plus, which offered more than 100 target patterns per line and allowed us to program the shape of a target by bit-mapping. The nominal time to track a full line of targets, assuming perfect tracking, was $1.7 \mathrm{sec}$. A 15-pixel target height allowed flexibility in target design. The time required for the raster to scan a single line was about $650 \mu \mathrm{sec}$, negligible relative to the speed of pursuit, leaving an off-time of about $16 \mathrm{msec}$. Thus, one can consider the line of targets to be displayed instantaneously, at a repetition rate of $60 / \mathrm{sec}$ with a fast-decay phosphor. Dwell time was about $0.4 \mu \mathrm{sec}$ on each pixel. The display was black-on-white, so that the intensity of the pursued targets was constant, and was not strobed. Although only the background varied in brightness, simultaneous brightness induction was adequate to create a picket-fence illusion and sigmaOKN eye movements as easily as was a more conventional strobed display. Viewing distance was $32.5 \mathrm{~cm}$, resulting in a horizontal screen size of $30.6^{\circ}$ and a target size of 20 arc min. These parameters were held constant throughout the experiments, so that the design could concentrate on the relationship of pursuit to changes in the target patterns.

A Pascal program provided a moving dot just under the line of target figures. Velocity of the moving dot was $18 \% / \mathrm{sec}$, slow enough that saccade-free tracking without delay was possible with minimal practice (McHugh \& Bahill, 1985). It had been shown previously in this laboratory that saccade-free pursuit could track a target in interrupted real motion even at much lower temporal frequencies than that used here: subjects followed with smooth pursuit a point target that moved in a sawtooth pattern, consisting of vertical jumps alternating with constant-slope motions. Eye movements were recorded with an infrared photoelectric system. Subjects were successful in generating smooth pursuits despite the irregular motion of the target, until the sawtooth frequency was lowered to 3-5 Hz. The pursuit system was able to integrate the signal and generate a smooth movement to a periodic stimulus (Bridgeman, Mayer, \& Glenn, 1976).

\section{Procedure}

Practice effects required that we use naive subjects for data collection so that amount of practice could be limited and controlled. Subjects were first screened to measure their ability to see the picketfence illusion and to track smoothly across a screenful of identical 
targets. At the beginning of the experiment, a subject was seated in front of the computer and was informed about the picket-fence illusion and how it works. Each subject practiced sigma-OKN and seeing the illusion with the aid of the experimenter on a stimulus consisting of a set of 102 vertical-line stimuli.

The subjects were instructed to follow the moving dot as long as it was present, and when it disappeared to look to the center of the moving character above it. They were reminded of the instruction on each trial. They were not told about the structures of the stimuli to be presented. Since pilot experiments showed initial rapid improvement in tracking ability as the subjects internalized the tracking pattern and learned zero-latency pursuit (Bahill \& McDonald, 1983), all subjects received oculomotor practice before the experiments began. Training consisted of $1 \mathrm{~min}$ of continuous tracking, alternating scan directions, followed by a 30-sec rest and a second minute of practice.

For each trial, the moving dot was presented for 1-2 full cycles of sawtooth tracking and was continued further until pursuit reached approximately the 25th target; then it disappeared, and one of four possible results was recorded: the eyes stopped (1) when the pointer disappeared, (2) at the barrier where the pattern changed, (3) between the barrier and the end of the line of stimuli, or (4) at the end of the line. Only the latter result was considered to be a successful tracking through a barrier. When tracking broke down, the subjects either found themselves staring at a target near the barrier or experienced a blurring of the line of stimuli. For each stimulus pattern, the dot disappeared during leftward scans three times and during rightward scans three times in an alternating sequence. For each subject, a threshold was found: If a subject succeeded in tracking to the end of a line in two or more of the three trials with a given stimulus pattern and direction, the difference in stimuli across the barrier was considered to be below threshold.

Since the distances scanned were large and the tracking was fast, it was not difficult to determine where tracking ceased. The subjects could easily determine whether they were tracking accurately because of the disappearance of the picket-fence illusion when sigmaOKN broke down. In addition to this subjective measure, oculomotor behavior was verified with direct observation of eye position.

\section{Stimuli}

We tested four kinds of patterns, each intended to answer a different question about the features that are important to the pursuit system.

Accretion/deletion. At the border between the two patterns, a solid vertical line changed to a line with a pixel missing from its center. A series of patterns had progressively more missing pixels until only one pixel was left at the top and bottom of the line

\section{|||||||||||||||||||||||||||||||||||||||||||||||||||||||||||||||||||||||||||||||||||}

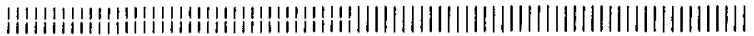

"

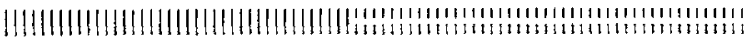

\section{|||||||||||||||||||||||||||||||||||||||||||||| \\ ||||||||||||||||||||||||||||||||||||||||}

Figure 1. Sample of the transition regions of the stimuli used in the missing pixels conditions. Top row: control condition, all pixels present. Second row: one pixel missing on the left side. Third and fourth rows: three pixels missing on the left and right sides, respectively. Fifth and sixth rows: 13 pixels missing on the left and right sides, respectively. Stimuli used in the experiment were 102 targets wide (i.e., wider than those illustrated here).

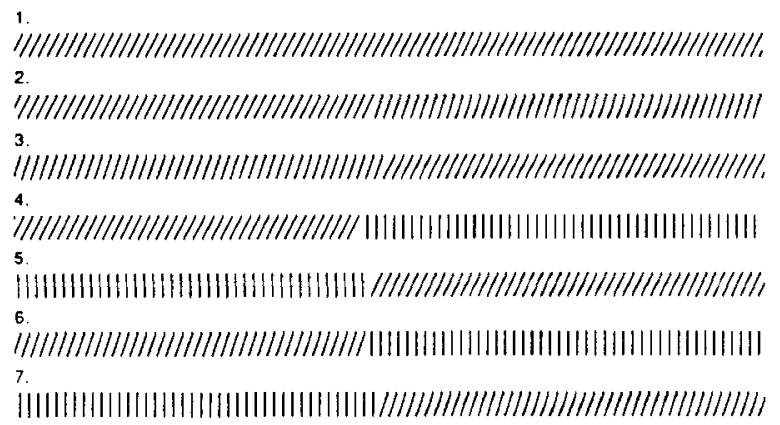

Figure 2. Sample of the transition regiens of the stimuli used in the orientation conditions. Top row: control condition, all orientations $26.5^{\circ}$ from vertical. Rows 2 and 3: transitions from $26.5^{\circ}$ to $18.4^{\circ}$. Rows 4 and 5: transitions from $26.5^{\circ}$ to $0^{\circ}$ (vertical). Rows 6 and 7: transitions from $0^{\circ}$ to $18.4^{\circ}$. Stimuli in the experiment were 102 targets wide.

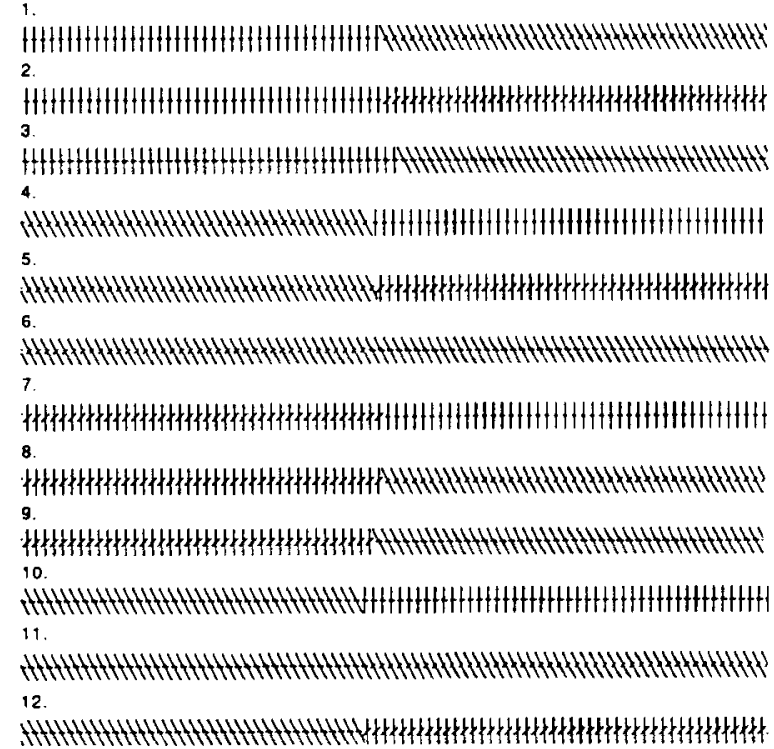

Figure 3. Stimulus set used for the experiments in changing the orientation of both a primary line and a "distractor" line. Subjects tracked about equally easily through all 12 patterns.

(Figure 1), resulting in 15 lines of stimuli. This stimulus has the advantage that it is easy to quantify the difference among the various patterns.

Orientation. Patterns were lines of various orientations, changing abruptly from one orientation to another at the border (Figure 2). There were seven lines of stimuli in this condition.

Distractors. Twelve rows of stimuli each consisted of two contours, one a straight line of a given slant and another intersecting line either orthogonal to the principal line or at another angle to it (Figure 3). The orientation of the principal line, the distractor, or both changed at the border. The subjects were not told of the distractor lines; they were told to fixate the center of the moving figure as before.

Scan width. With vertical-line targets, successive patterns had successively more targets missing from the center of the display until the gap was 21 targets wide (Figure 4). These will be called 


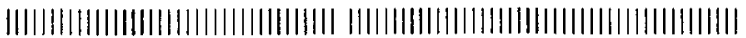

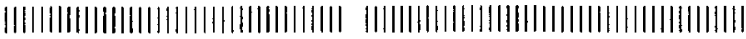
|||||||||||||||||||||||||||||| $\mid$

||||||||||||||||||||||||||||

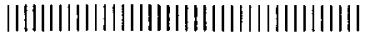

|||||||||||||||||||||||||||||||||||||

Figure 4. Sample of the transition regions in the tunnel experiments. Top row: one missing target. Second row: two missing targets. Third row: nine missing targets. Fourth row: 20 missing targets. Position of the tunnel is varied slightly around a symmetrically centered location.

"tunnel" experiments, using the terminology of Collewijn, Curio, and Grüsser (1982). A small amount of "jitter" was added to the location of the gap near the center of the line.

\section{RESULTS}

Twenty subjects participated in the accretion/deletion series and in the orientation series. The first pattern was a control with continuous vertical lines and no barrier, which all subjects tracked successfully. Below the threshold stimulus change, sigma-OKN through the barrier would succeed; above the threshold change, the subject would fail to track through the barrier. The switch from failure to success usually occurred between one number of missing pixels and the next, with no failures at smaller numbers of missing pixels. Thus, the psychophysical curves were quite steep for a given subject and stimulus.

\section{Accretion/Deletion}

As the number of missing pixels was increased at the border, making the left and right sides more and more dissimilar to each other, an increasing number of subjects failed to track through the border. This result validates the method, showing that psychophysical curves can be obtained from the picket-fence illusion to quantify the degree of similarity of two patterns to the pursuit-eyemovement control system.

For each pattern, the mean thresholds for successful sigma-OKN to the right and to the left were compared. It had been hypothesized that rightward tracking might be less smooth for these internally generated pursuit movements because of a possible intrusion of habitual reading saccades in this direction. The thresholds for rightward sigma-OKN were not significantly different from the thresholds for leftward sigma-OKN (Figure 5) with any pattern, however (maximum $\chi^{2}=1.67$, n.s.). Similarly, deletion was no different from accretion (maximum $\chi^{2}=0.96$, n.s.).

Half of the subjects were able to track through the largest barrier, where 13 of the 15 pixels were deleted from the target, leaving only a single pixel to define each end of the previously continuous line. For these subjects, $13^{\circ}$ was taken as the threshold because removing the final two pixels would, of course, make tracking impossible. This "ceiling effect" complicates the interpretation of the accretion/deletion results. The data do show that many subjects can track through even a large change in the stimulus, however, as long as the size and orientation of the targets remain constant.

\section{Orientation}

The threshold for successful pursuit was not significantly different for leftward and rightward tracking (Figure 6).

More than half of the subjects were able to track through the largest orientation difference, however, so that the orientation differences in degrees in Figure 6 underestimate the threshold orientation difference that prevented sigma-OKN. Larger orientation differences could not be presented for technical reasons. Larger differences might have provided a stronger barrier to sigma-OKN but would have necessitated a change in the shape of the target window, and it would have been necessary to display fewer targets on our screen. Thus, the conditions of stimulation at various orientation differences would not have

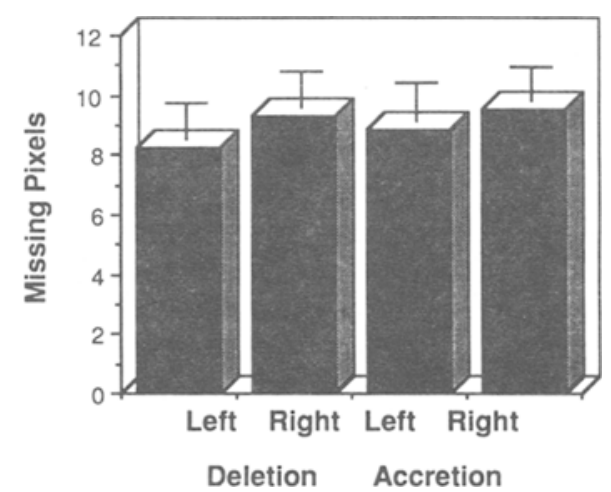

Figure 5. Mean number of pixels that could be omitted from the center of a target before pursuit eye movements ceased. Stimuli used in this condition are shown in Figure 1. Error bars extend one between-subjects standard error above the mean. The figure summarizes psychophysical curves from 20 subjects. Left and right refer to direction of pursuit eye movement on the scan where the tracking spot was extinguished. In the deletion series, the eye tracked from complete targets to targets with the indicated number of pixels omitted; in the accretion series, the eye tracked from incomplete to complete targets.

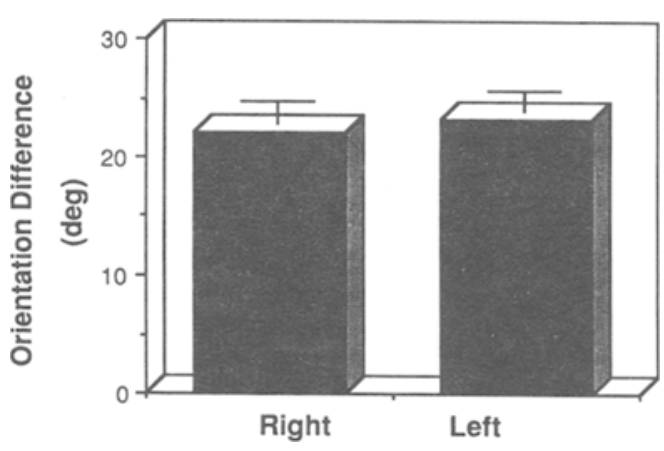

Figure 6. Number of degrees difference in orientation of straight bars at a transition where target orientation changes abruptly at the center of the screen. Stimuli used in this condition are shown in Figure 2. The figure summarizes psychophysical curves from $\mathbf{2 0}$ subjects. Display format as in Figure 5 . 
been comparable. A plot of the number of subjects able to track through each barrier, however, shows a linear relationship between number of subjects and orientation difference in the stimuli (Figure 7). The curves indicate that the median subject could track through an orientation difference of $31.8^{\circ}$. This is an extrapolation from the data in Figure 7, but only a slight one, because the predicted median orientation for successful tracking is only about $5^{\circ}$ from the last data point, and the linear regression line on which it is based accounts for $92 \%$ of the variance in the between-subjects data.

\section{Distractors}

In this series, 27 subjects were tested on scan width and on the ability to track through barriers with orthogonal or nonorthogonal distractor lines. Nine of the subjects had also participated in the two conditions above.

Addition of a second line to the pursued target, no matter what the second line's orientation, had little effect on sigma-OKN. Changes in the orientation of the distractor at the barrier did not affect sigma-OKN, perhaps because the principal line provided a strong correlation between the targets on the two sides of the barrier and the shorter distractor line was inadequate to interfere with tracking of the principal line. Despite the ability to track through the barriers, the changes in the stimuli were clearly visible to all observers.

Why could subjects track through changes in distractors even though in the pilot experiments a 1-to-x boundary was difficult? Perhaps a change in the topology of the characters is more important than small changes in orientations of parts, as long as topology remains constant.

\section{Scan Width}

When sigma-OKN was successful, subjects often noted the appearance of a tunnel through which the obscured

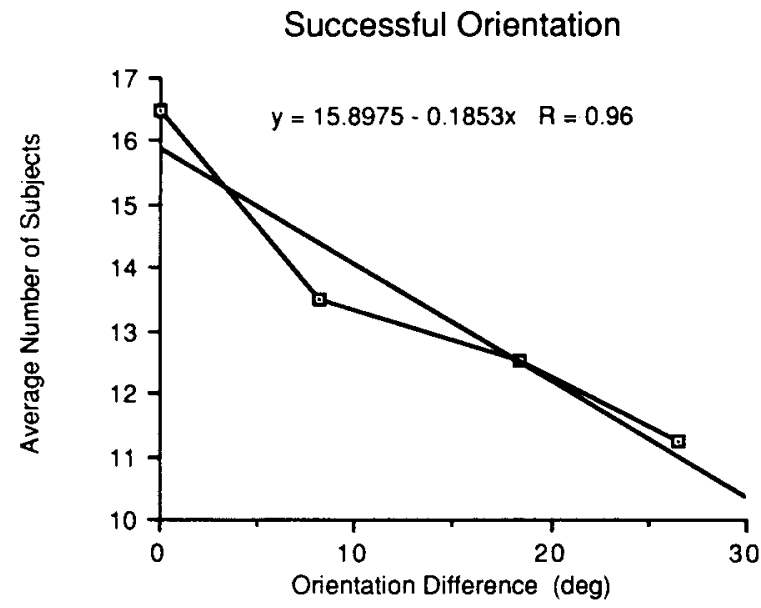

Figure 7. Number of subjects completing pursuit through an abrupt change in target orientation at the center of the screen. Stimuli used in this condition are shown in Figure 2. The figure summarizes psychophysical curves from 20 subjects. The line through the data is a least-squares linear fit. Left-to-right and right-to-left tracking, which were nearly identical, are collapsed at each orientation.

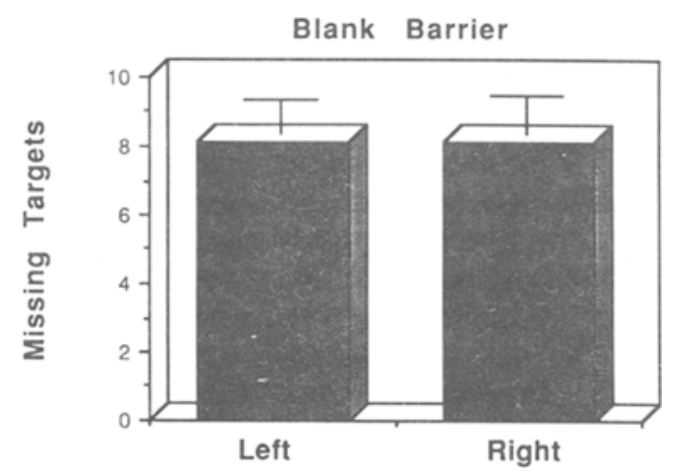

Figure 8. Mean number length of a tunnel of missing targets through which the picket-fence illusion continued. The number of missing targets is plotted on the abscissa. Each missing target represents 16.7 msec of pursuit without a stimulus at the center of the fovea. Stimuli used in this condition are shown in Figure 4. Display format as in Figure 5 . The figure summarizes psychophysical curves from 29 subjects.

line seemed to be moving. Continuation of the picket-fence illusion on both sides of the tunnel indicated a continuation of sigma-OKN pursuit movements. With this stimulus array, subjects tracked despite the presence of a low spatial-frequency component of the stimulus that remained fixed relative to the screen. The mean threshold for recovering the picket-fence illusion on the other side of a tunnel (and maintaining sigma-OKN through the tunnel) was 8.125 missing targets, resulting in $135 \mathrm{msec}$ without a consistent foveal target (Figure 8). None of the subjects was able to track through the maximum barrier of 21 missing targets.

This condition extends a result of Collewijn et al. (1982), who found successful tracking through tunnels of five targets. Tunnels larger than this were not tried. Collewijn et al. used a larger intertarget distance $\left(0.75^{\circ}\right)$, a larger target $\left(0.5^{\circ} \mathrm{high}\right)$, and a slower flicker rate $(15 \mathrm{~Hz})$ than those used in the present experiment. Thus, their subjects could track through a tunnel of at least $333 \mathrm{msec}$, compared to $135 \mathrm{msec}$ of the median subject in the present study. Under the faster strobe conditions used here, a 333-msec tunnel corresponds to 20 missing targets; only 2 of the 27 subjects maintained the motion illusion through a tunnel that long in the rightward direction, and 1 in the leftward direction. The frequency of sampling, rather than the time constant of the pursuit oculomotor system, seems to be the critical limiting factor in determining the duration of a penetrable tunnel.

\section{DISCUSSION}

The ability of subjects to track through a change in stimulus-target characteristics did not depend on perceivable differences between the targets on the two sides of the barrier. In every case, differences between the patterns of the targets were well above perceptual threshold before sigma-OKN began to degrade significantly. Instead, breakdown in sigma-OKN seemed to correlate with 
the characteristics of visual receptive fields and of the pursuit system itself.

In the orientation series, the sigma-OKN threshold in the median subject was not at the smallest detectable orientation difference, but at an orientation difference of $31.8^{\circ}$. This number can be used to estimate the bandwidth of the orientation-sensitive channels that drive the pursuit system. According to Schiller, Finlay, and Volman (1976), the bandwidth of orientation-sensitive channels in striate cortex is $30^{\circ}$, the modal value for their "S-type" neurons. Thus, sigma-OKN broke down at an orientation difference at which the modal receptive field could not code the stimuli on both sides of the barrier.

This interpretation neglects the variations in performance between subjects. An explanation that suffices for a subject who ceased tracking at an orientation difference of $30^{\circ}$, for instance, will be unsatisfactory for a subject who ceased at $20^{\circ}$. Correspondingly, though, the orientation bandwidths of cortical neurons also vary widely, from about $10^{\circ}$ to more than $120^{\circ}$ (Schiller et al., 1976). If this interpretation is correct, one would expect wide individual differences in bandwidths of orientation-tuned receptive fields in humans. This result has been found in human orientation channels (Blakemore \& Nachmias, 1971; Thomas \& Gille, 1979). Psychophysical channel bandwidth was estimated in the latter study by measuring both detection and identification of orientation differences. The edge of a band was reached when the ratio of identification to detection approached 1 . Half-amplitude bandwidths were $10^{\circ}$ to $20^{\circ}$, less than the present estimate from the pursuit-interruption method.

We can conclude that the pursuit system takes its orientation information from an earlier state of visual processing than does perception, a stage at which channels have not yet been tuned to bandwidths narrower than those of striate cortex neurons. This conclusion is consistent with Robinson's (1981) point that the pursuit system has little time to act and must obtain its information from the earliest possible stages of visual processing. Lisberger, Morris, and Tychsen (1987) reviewed physiological evidence that inputs for pursuit come from several levels, beginning at V1 (striate cortex).

Adler and Grüsser (1982) have shown that sigma-OKN can be maintained by a random-dot stereogram pattern that excites the cyclopean retina without being visible to either eye. Thus, the stimulus to pursuit must be generated by binocular mechanisms located beyond Layer IV, the principal input layer of striate cortex.

The failure to find significant differences in success of tracking among the various distractor conditions might be attributable to a similar receptive-field interpretation. The idea rests on the existence of stages of visual processing in which common features in all of the distractor stimuli (Figure 3) are coded by a common mechanism. The presence of an intersection between two line segments imparts a high degree of structural similarity to the patterns, regardless of changes in their orientations. If recep- tive fields sensitive to this structure can drive the pursuit system, tracking could continue through the barriers.

In the tunnel experiments, threshold scan width can be compared to the temporal integration ability of the pursuit system measured with other methods. Mitrani and Dimitrov (1978) found that after a target disappeared, pursuit movements continued at the rate of target motion for about $300 \mathrm{msec}$. However, the duration of the open-loop drift was longer when the duration of target motion was shorter, implying that the pursuit system's information about target motion was less precise with short durations. Thus, it required more time to determine that motion was no longer present when the stimulus sample was shorter.

The subjects in the present study tracked for a relatively short time; the stimulus was visible for only $750 \mathrm{msec}$ before the subject encountered the tunnel, which, according to Mitrani and Dimitrov (1978), should yield an increase of about $33 \%$ in open-loop drift. Nevertheless, mean tracking time in the present experiment was only $135 \mathrm{msec}$. Two differences between the present stimulus array and Mitrani and Dimitrov's may explain the differences in duration of tracking without a foveal target. First, their subjects were tracking on a uniform screen, whereas the subjects in the present study sat in a contour-rich visual environment. Second, the stimuli on both sides of the present barrier remained visible and moved with each flash to provide a stimulus to steady fixation. Both of these factors should reduce drift time compared to open-loop conditions.

The existence of sigma-OKN tracking depends upon an incorrect solution to the correspondence problem in apparent motion (Ullman, 1979). During tracking, the pursuit system interprets each successive target as belonging with the adjacent target on the previous raster scan. The spatiotopically correct solution, that each target flashes in an egocentrically fixed location, is apparent only at the borders of the tunnel. In the present experiment, the existence of other contours in the field makes it easier to utilize the spatiotopic information at the borders.

The threshold for ability to track through the tunnel did not depend on the similarity of targets to the input of the pursuit control system; rather, it relied on the ability of the system to maintain smooth eye movements in the absence of a smoothly moving foveal target. Other experiments have also shown a relatively long integration time in pursuit control. The eye can pursue a target moving with a vertical sawtooth component and a linear horizontal component, and can maintain horizontal saccade-free pursuit with a sawtooth period of up to $370 \mathrm{msec}$ (Bridgeman et al., 1976). Thus, pursuit of the picket-fence illusion through a barrier in the present experiment ceased at less than half of the duration of the human subject's ability to integrate continuously visible targets with vertical error in the sawtooth experiments.

The experiments demonstrate that maintenance of pursuit depends upon the formation of an internal template of the image being tracked; if the stimulus varies too 
greatly from the characteristics of this template, tracking ceases. Isolated stimuli always contain low spatialfrequency components that can form part of the template; a new isolated stimulus will contain similar low-frequency components independent of changes in its high spatialfrequency components, brought about by changes in the internal geometry of the stimulus. The pursuit-interruption method introduced here allows a complete change in the template, whereas more conventional methods do not. The present method also allows a glimpse at properties of an earlier stage of visual processing than that available to perception.

\section{REFERENCES}

Adler, B., Collewijn, H., Curio, G., Grüsser, O.-J., Pause, M., SChreiter, U., Weiss, L. (1981). Sigma-movement and sigmanystagmus: A new tool to investigate the gaze pursuit system and visual movement perception in man and monkey. Annals of the New York Academy of Sciences, 374, 284-302.

ADLER, B., \& GRüsSER, O.-J. (1982). Sigma-movement and optokinetic nystagmus elicited by stroboscopically illuminated stereopatterns. $E x$ perimental Brain Research, 47, 353-364.

BAhill, A. T., \& MCDonALD, J. (1983). Smooth pursuit eye movements in response to predictable target motions. Vision Research, 23, 1573-1578.

Behrens, F., \& GRüsSER, O.-J. (1978). Bewegungswahrnehmung und Augenbewegungen bei Flickerbelichtung unbewegter visueller Muster. In G. Kommerell (Ed.), Augenbewegungsstörungen Neurophysiologie und Klinik (pp. 273-283). Munich: Bergmann.

Blakemore, C., \& NaChMIAS, J. (1971). The orientation specificity of two visual aftereffects. Journal of Physiology, 213, 157-174.

Bridgeman, B., Kirch, M., \& Sperling, A. (1981). Segregation of cognitive and motor aspects of visual function using induced motion. Perception \& Psychophysics, 29, 336-342.

Bridgeman, B., Mayer, M. J., G Glenn, L. (1976). Figure distortion accompanying pursuit eye movements. Vision Research, 16, 431-433.

Collewun, H., Curio, G., \& Grüsser, O.-J. (1982). Spatially selec- tive visual attention and generation of eye pursuit movements. $\mathrm{Hu}$ man Neurobiology, 1, 129-139.

Deubel, H., Findlay, J., Jacobs, A., Brogan, D. (1988). Saccadic eye movements to targets defined by structure differences. In G. Lüer, U. Lass, \& J. Shallo-Hoffman (Eds.), Eye movement research: Physiological and psychological aspects (pp. 107-145). Toronto: Hogrefe.

HeYwood, S. (1973). Pursuing stationary dots: Smooth eye movements and apparent movement. Perception, 2, 181-195.

Kolers, P. A., \& Pomeranz, J. R. (1971). Figural change in apparent motion. Joumal of Experimental Psychology, 87, 99-108.

LAMONTAGNE, C. (1973). A new experimental paradigm for the investigation of the secondary system of human visual motion perception. Perception, 2, 167-180.

Lisberger, S., Morris, E., \& Tychsen, L. (1987). Visual motion processing and sensory-motor integration for smooth pursuit eye movements. Annual Review of Neurosciences, 10, 97-129.

McHugh, D. E., BAHILL, A. T. (1985). Learning to track predictable target waveforms without a time delay. Investigative Ophthalmology \& Visual Science, 26, 932-937.

Mitrani, L., \& Dimitrov, G. (1978). Pursuit eye movements of a disappearing moving target. Vision Research, 18, 537-539.

RoBINSON, D. A. (1981). Control of eye movements. In J. Brookhart \& V. Mountcastle (Eds.), Handbook of physiology: Vol. 1: II.2. Motor control (pp. 1275-1320). Bethesda, MD: American Physiological Society.

SChIller, P. H., Finlay, B. L., \& Volman, S. F. (1976). Quantitative studies of single-cell properties in monkey striate cortex: 2. Orientation specificity and ocular dominance. Journal of Neurophysiology, 39, 1334-1351.

STOPER, A. E. (1973). Apparent motion of stimuli presented stroboscopically during pursuit movement of the eye. Perception \& Psychophysics, 13, 201-211.

Thомas, J. P., \& Glle, J. (1979). Bandwidths of orientation channels in human vision. Joumal of the Optical Society of America, 69, 652-660.

Ullman, S. (1979). The interpretation of visual motion. Cambridge, MA: MIT Press.

WONG, E., MACK, A. (1980). Saccadic programming and perceived location. Acta Psychologica, 48, 123-131.

(Manuscript received August 19, 1988; revision accepted for publication March 10, 1989.) 